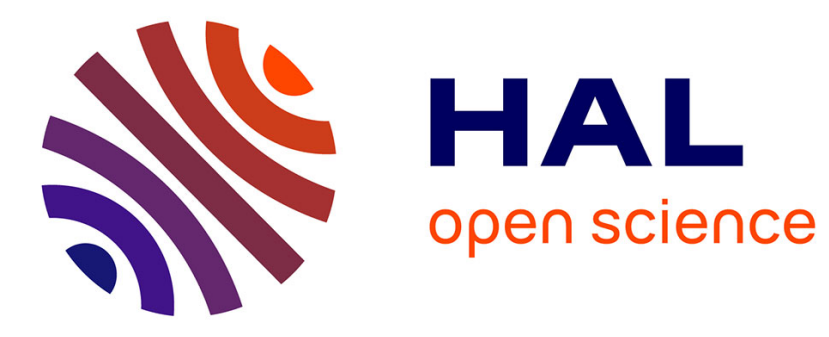

\title{
Energy-based nonlinear control for a quadrotor rotorcraft
}

Laura Muñoz, Omar Santos, Pedro Castillo, Isabelle Fantoni

\section{To cite this version:}

Laura Muñoz, Omar Santos, Pedro Castillo, Isabelle Fantoni. Energy-based nonlinear control for a quadrotor rotorcraft. American Control Conference (ACC 2013), Jun 2013, Washington, DC, United States. pp.1177-1182. hal-00927067

\section{HAL Id: hal-00927067 https://hal.science/hal-00927067}

Submitted on 10 Jan 2014

HAL is a multi-disciplinary open access archive for the deposit and dissemination of scientific research documents, whether they are published or not. The documents may come from teaching and research institutions in France or abroad, or from public or private research centers.
L'archive ouverte pluridisciplinaire HAL, est destinée au dépôt et à la diffusion de documents scientifiques de niveau recherche, publiés ou non, émanant des établissements d'enseignement et de recherche français ou étrangers, des laboratoires publics ou privés. 


\title{
Energy-based nonlinear control for a quadrotor rotorcraft
}

\author{
L. E. Muñoz ${ }^{1}$ and O. Santos ${ }^{2}$ and P. Castillo ${ }^{1}$ and I. Fantoni ${ }^{1}$
}

\begin{abstract}
A sub-optimal feedback control to stabilize a quadrotor vehicle is introduced in this paper. The approach makes use of the passivity property and the dynamic programming theory. The control design is based on the proposition of a Lyapunov function which has been defined as the energetic function for the system and, at the same time, is an approximation of the Bellman function associated with the optimal problem for nonlinear systems. To validate the control strategy, various simulations have been carried out.
\end{abstract}

\section{INTRODUCTION}

The development of Unmanned Aerial Vehicles (UAV's), especially the quadrotor configuration, has generated great interest in the control community in the last few decades. This vehicle is based on a VTOL (Vertical Take-Off and Landing) concept and it is extensively used to develop control laws. The particular interest of the research community for the quadrotor design can be linked to two main advantages over comparable VTOL UAVs, such as helicopters. First, quadrotors do not require complex mechanical control linkages for rotor actuation, relying instead on fixed pitch rotors and using variation in motor speed for vehicle control. This simplifies both the design and maintenance of the vehicle. Second, the use of four rotors ensures that individual rotors are smaller in diameter than the equivalent main rotor on a helicopter, relative to the airframe size. The quadrotor configuration will therefore be easier to handle and possesses a higher maneuverability.

Previous works in quadrotor vehicles have often ignored gyroscopic, Coriolis and aerodynamic effects (like wind) [1], [2], [3]. At slow velocities, such as hovering, this is indeed a reasonable assumption. However, even at moderate velocities, the impact of these effects resulting from variation in air speed is significant. Therefore, it is important to take into account these terms in the mathematical models when proposing control strategies that are robust to bounded variations of these effects.

Although many control laws have been proposed in the literature for quadrotor UAVs [4], [5], [6], [7], the passivity property of these vehicles has not been extensively explored. Nevertheless in [8], passive components of the quadrotor aircraft model have allowed to develop independent simple PD controllers, taking into account the limitation of control range and permitting to provide stability property.

*This work was supported by CONACYT

${ }^{1}$ L. E. Muñoz, P. Castillo and I. Fantoni are with Heudiasyc Lab. UMR 7253, Université de Technologie de Compiègne, 60200 Compiègne, France, (lmunozhe, castillo, ifantoni) @hds.utc.fr

${ }^{2} \mathrm{O}$. Santos is with the Universidad Autónoma del Estado de Hidalgo, Pachuca, Hidalgo, México, omarj@uaeh.edu.mx
Interesting features of passive systems are that there is no internal creation of energy and that they have stable (in the sense of Lyapunov) natural equilibrium configurations, which correspond to their minimum energy. This work focuses on proposing an energy-based nonlinear control law to stabilize a quadrotor flying vehicle when using its passivity property. Furthermore, the optimization of energy in this type of vehicles is fundamental. Optimal control provides a potentially useful methodology to design nonlinear control laws $u=u(x)$ which give the appropriate action $u$ for any state $x$. Several strategies based on optimal control have been proposed for quadrotor vehicles. For example in [9], a linear $H_{\infty}$ outer control for quadrotor helicopter with plant uncertainty combined with a robust feedback linearization inner controller have been developed and validated only in simulations. Similarly in [10], a constrained finite-time optimal controller for the attitude control of a quadrotor helicopter under wind gusts and physical constraints has been presented.

In the proposed paper, the methodology will be based on a combination of an energy-based and optimal control approaches applied on the nonlinear quadrotor system. The dynamic programming provides a way to find globally optimal control laws, given in a step. Then, we take advantage of the passivity property of the system in order to establish a control law based on Lyapunov theory stability and the Dynamic Programming approach. By using Dynamic Programming, we synthesized a suboptimal control using the passivity property for the quadrotor aircraft. This approach was proposed to underactuated systems in [11] and experimentally tested in a pendubot in [12]. In our contribution, the extension of this control strategy is successfully applied to UAVs. The proposed control method involves two expressions: an energy-based term and a nonlinear feedback term. The first one uses the passivity property present in the quadrotor model and the second one uses the advantage of state feedback. Several simulations are carried out to validate the proposed control scheme. In addition, the performance of this algorithm is compared with respect to a linear feedback controller (without perturbations) and with a Linear Quadratic Regulator (LQR) algorithm (with wind disturbances). In both cases the proposed controller has a better behavior and it can be expressed that its passivity part gives a robustness component when external perturbations appear.

The outline of the paper is the following: in section II we describe a brief survey of the mathematical representation of the quadrotor vehicle and the main concepts of the optimal control and dynamic programming. The energy-based non- 
linear control law is developed in section III. To validate the performance of the proposed control scheme, simulations are carried out and in section IV, the main results are illustrated. Finally, conclusions and future work are discussed in section V.

\section{BACKGROUND}

\section{A. Mathematical description of a quadrotor}

Quadrotor helicopters represent a famous rotorcraft concept for UAV platforms. The vehicle consists of four rotors in total, with two pairs of counter-rotating, fixed-pitch blades located at the four corners of the aircraft, see Figure 1.

The Lagrange-Euler dynamic equations in terms of $\mathbf{q}=$ $\left[\begin{array}{ll}\xi & \eta\end{array}\right]^{T}$ can be written, in general form, as follows [13]

$$
\mathbf{M}(\mathbf{q}) \ddot{\mathbf{q}}+\mathbf{C}(\mathbf{q}, \dot{\mathbf{q}}) \dot{\mathbf{q}}+\mathbf{G}(\mathbf{q})=\mathbf{Q}_{\tau}
$$

with

$$
\begin{gathered}
\mathbf{M}=\left[\begin{array}{cc}
m \mathbf{I}_{3 \times 3} & \mathbf{0}_{3 \times 3} \\
\mathbf{0}_{3 \times 3} & \mathbf{J}_{\eta} 3 \times 3
\end{array}\right] ; \quad \mathbf{C}=\left[\begin{array}{cc}
\mathbf{0}_{3 \times 3} & \mathbf{0}_{3 \times 3} \\
\mathbf{0}_{3 \times 3} & \mathbf{C}_{\eta} \times 3
\end{array}\right], \\
\mathbf{G}=\left[\begin{array}{c}
\mathbf{G}_{\mathbf{g}} \\
\mathbf{0}
\end{array}\right] ; \quad \mathbf{Q}_{\tau}=\left[\begin{array}{c}
\mathbf{f} \\
\tau
\end{array}\right],
\end{gathered}
$$

where $\mathbf{I}$ denotes the identity matrix, $\xi=[x, y, z]^{T}$ describes the position of the center of mass of the helicopter and $\eta=\left[\begin{array}{l}\psi \\ \theta, \phi\end{array}\right]^{T}$ represents the Euler angles (yaw, pitch and roll, respectively). $\mathbf{G}_{\mathbf{g}}=\left[\begin{array}{lll}0 & 0 & \mathrm{mgz}\end{array}\right]^{T}$ with $z$ means the quadrotor altitude, $m$ indicates the mass of the quadrotor and $g$ is the acceleration due to gravity. $\mathbf{C}_{\eta}$ defines the Coriolis matrix whilst $\mathbf{J}_{\eta}$ describes the inertia matrix for the full rotational kinetic energy of the helicopter. In addition, f and $\tau$ represent respectively the translational forces and the torque vectors. Moreover, their expressions are given as

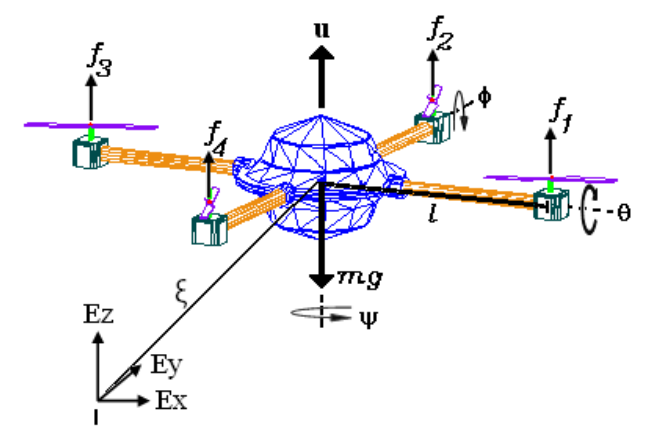

follows

$$
\begin{aligned}
\mathbf{f} & =\left[\begin{array}{ll}
-s_{\theta} & u \\
c_{\theta} s_{\phi} & u \\
c_{\theta} c_{\phi} & u
\end{array}\right] \\
\tau & =\left[\begin{array}{l}
\tau_{\psi} \\
\tau_{\theta} \\
\tau_{\phi}
\end{array}\right]=\left[\begin{array}{c}
\sum \tau_{M_{j}} \\
\left(f_{3}-f_{1}\right) l \\
\left(f_{2}-f_{4}\right) l
\end{array}\right] \\
\mathbf{J}_{\eta} & =\left[\begin{array}{lll}
J_{\eta_{11}} & J_{\eta_{12}} & J_{\eta_{13}} \\
J_{\eta_{12}} & J_{\eta_{22}} & 0 \\
J_{\eta_{13}} & 0 & J_{\eta_{33}}
\end{array}\right] \\
\mathbf{C}_{\eta} & =\left[\begin{array}{lll}
C_{\eta_{11}} & C_{\eta_{12}} & C_{\eta_{13}} \\
C_{\eta_{21}} & C_{\eta_{22}} & C_{\eta_{23}} \\
C_{\eta_{31}} & C_{\eta_{32}} & C_{\eta_{33}}
\end{array}\right]
\end{aligned}
$$

where $u=\sum f_{i}$ means the main thrust, $\tau$ indicates the generalized torques (yaw, pitch and roll moments). $f_{j}$ and $\tau_{M_{j}}$ are the forces and the moments produced by the motor $j, j: 1,2,3,4$, and $l$ the distance between the motor $j$ and the center of gravity of the helicopter. By simplicity, we use $c_{\theta}$ and $s_{\theta}$ for $\cos (\theta)$ and $\sin (\theta)$ respectively.

In addition,

$$
\begin{aligned}
& J_{\eta_{11}}=I_{x x} s_{\theta}^{2}+I_{y y} c_{\theta}^{2} s_{\phi}^{2}+I_{z z} c_{\theta}^{2} c_{\phi}^{2} ; J_{\eta_{12}}=c_{\theta} c_{\phi} s_{\phi}\left(I_{y y}-I_{z z}\right) \\
& J_{\eta_{13}}=-I_{x x} s_{\theta} ; J_{\eta_{22}}=I_{y y} c_{\phi}^{2}+I_{z z} s_{\phi}^{2} ; J_{\eta_{33}}=I_{x x} \\
& C_{\eta_{11}}=I_{x x} \dot{\theta} s_{\theta} c_{\theta}+I_{y y}\left(-\dot{\theta} s_{\theta} c_{\theta} s_{\phi}^{2}+\dot{\phi} c_{\theta}^{2} s_{\phi} c_{\phi}\right) \\
& -I_{z z}\left(\dot{\theta} s_{\theta} c_{\theta} c_{\phi}^{2}+\dot{\phi} c_{\theta}^{2} s_{\phi} c_{\phi}\right) \\
& C_{\eta_{12}}=I_{x x} \dot{\theta} s_{\theta} c_{\theta}-I_{y y}\left(\dot{\theta} s_{\theta} s_{\phi} c_{\phi}+\dot{\phi} c_{\theta} s_{\theta}^{2}-\dot{\phi} c_{\theta} c_{\phi}^{2}+\dot{\psi} s_{\theta} c_{\theta} s_{\phi}^{2}\right) \\
& +I_{z z}\left(\dot{\phi} c_{\theta} s_{\phi}^{2}-\dot{\phi} c_{\theta} c_{\phi}^{2}-\psi s_{\theta} c_{\theta} c_{\phi}^{2}+\dot{\theta} s_{\theta} s_{\phi} c_{\phi}\right) \\
& C_{\eta_{13}}=-I_{x x} \dot{\theta} c_{\theta}+I_{y y} \dot{\psi} c_{\theta}^{2} s_{\phi} c_{\phi}-I_{z z} \dot{\psi} c_{\theta}^{2} s_{\phi} c_{\phi} \\
& C_{\eta_{21}}=-I_{x x} \dot{\psi} s_{\theta} c_{\theta}+I_{y y} \dot{\psi} s_{\theta} c_{\theta} s_{\phi}^{2}+I_{z z} \dot{\psi} s_{\theta} c_{\theta} c_{\phi}^{2} \\
& C_{\eta_{22}}=-I_{y y} \dot{\phi} s_{\phi} c_{\phi}+I_{z z} \dot{\phi} s_{\phi} c_{\phi} \\
& C_{\eta_{23}}=I_{x x} \dot{\psi} c_{\theta}+I_{y y}\left(-\dot{\theta} s_{\phi} c_{\phi}+\psi c_{\theta} c_{\phi}^{2}-\psi c_{\theta} s_{\phi}^{2}\right) \\
& +I_{z z}\left(\dot{\psi} c_{\theta} s_{\phi}^{2}-\psi c_{\theta} c_{\phi}^{2}+\theta s_{\phi} c_{\phi}\right) \\
& C_{\eta_{31}}=-I_{y y} \dot{\psi} c_{\theta}^{2} s_{\phi} c_{\phi}+I_{z z} \dot{\psi} c_{\theta}^{2} s_{\phi} c_{\phi} \\
& C_{\eta_{32}}=-I_{x x} \psi c_{\theta}+I_{y y}\left(\dot{\theta} s_{\phi} c_{\phi}+\dot{\psi} c_{\theta} s_{\phi}^{2}\right. \\
& \left.-\dot{\psi} c \phi c_{\phi}^{2}\right)-I_{z z}\left(\dot{\psi} c_{\theta} s_{\theta}^{2}-\dot{\psi} c_{\theta} c_{\phi}^{2}+\dot{\theta} s_{\phi} c_{\phi}\right) \\
& C_{\eta_{33}}=0
\end{aligned}
$$

\section{B. Optimal control and dynamic programming}

It is well known that in an optimal control problem for a dynamic system, the task is to find an admissible control $\mathbf{u}^{*}$ generating the corresponding state trajectory $\mathbf{x}^{*}$ such that the cost functional

$$
J=\int_{0}^{\infty} f_{0}(\mathbf{x}, \mathbf{u}) d t
$$

is minimized. In the above equation, $f_{0}(\cdot)$ is a positive definite specified function. Let be the nonlinear system

$$
\dot{\mathbf{x}}(t)=f(\mathbf{x}, \mathbf{u})
$$

Without loss of generality, we suppose that system (3) has the equilibrium point zero.

Fig. 1. Quadrotor scheme 
Theorem 1: [14] If there exists a positive definite function $V^{*}(\mathbf{x}(t))$, which is continuously differentiable and satisfies

$$
\left.\frac{d V^{*}(\mathbf{x}(t))}{d t}\right|_{(3)}+f_{0}\left(\mathbf{x}^{*}, \mathbf{u}^{*}\right)=0
$$

then $\mathbf{u}^{*}$ is the optimal control.

Theorem 2: [15] Let $H(\mathbf{x}, \mathbf{u})$ be a scalar single valued function of the variables $\mathbf{x}$ and $\mathbf{u}$. Let $\frac{\partial^{2}\left(H\left(\mathbf{x}, \mathbf{u}^{*}\right)\right)}{\partial \mathbf{u}^{2}}$ exists and be bounded and continuous. Also assume that

$$
\frac{\partial H^{*}(\mathbf{x}, \mathbf{u})}{\partial \mathbf{u}}=0
$$

and

$$
\frac{\partial^{2}\left(H\left(\mathbf{x}, \mathbf{u}^{*}\right)\right)}{\partial \mathbf{u}^{2}}>0
$$

then $\mathbf{u}^{*}$ is a local minimum.

Notice that in Theorem 1, the Bellman functional $V^{*}(\mathbf{x}(t))$ is proposed to obtain the optimal control $\mathbf{u}^{*}(t)$ and to solve the optimal parameters of the Hamilton-Jacobi-Bellman (HJB). However, for nonlinear systems, it is not a trivial problem to solve the HJB equation given by (4). One problem is to propose or construct the Bellman equation $V^{*}(\mathbf{x}(t))$. In this paper, we proposed an approximation of the Bellman equation, using the passivity property and an energetic function, which is simultaneously, a Lyapunov function.

\section{ENERGY-BASED CONTROL LAW}

The goal of the quadratic optimal control algorithm is to find the feedback control, $\mathbf{u}$, which minimizes the following performance index

$$
\mathbf{J}=\frac{1}{2} \int_{0}^{\infty}\left(\overline{\mathbf{x}}^{T} Q \overline{\mathbf{x}}+\mathbf{u}^{T} R \mathbf{u}\right) d t
$$

where $Q=Q^{T}, Q>0$ and $R$ is a symmetric and strictly positive definite matrix and $\overline{\mathbf{x}}=\left[\begin{array}{ll}\overline{\mathbf{x}}_{1} & \overline{\mathbf{x}}_{2}\end{array}\right]^{T}$, where $\overline{\mathbf{x}}_{1}=\overline{\mathbf{q}}$ and $\overline{\mathbf{x}}_{2}=\dot{\overline{\mathbf{q}}}$, subject to the nonlinear model

$$
M(\overline{\mathbf{q}}) \ddot{\overrightarrow{\mathbf{q}}}+C(\overline{\mathbf{q}}, \dot{\overline{\mathbf{q}}}) \dot{\overline{\mathbf{q}}}+G(\overline{\mathbf{q}})=\mathbf{u}
$$

with $\overline{\mathbf{q}}=\mathbf{q}-\mathbf{q}_{\mathbf{e q}}, \dot{\overline{\mathbf{q}}}=\dot{\mathbf{q}}-\dot{\mathbf{q}}_{\text {eq }}$ where $\mathbf{q}_{\text {eq }}$ represents the desired state vector and $\mathbf{u}$ indicates the control input vector.

Observe that system (8) could be easily rewritten in the standard form (3):

$$
\begin{aligned}
\dot{\overline{\mathbf{x}}}= & \mathbf{f}(\overline{\mathbf{x}}, \mathbf{u}) \\
= & {\left[\begin{array}{c}
\overline{\mathbf{x}}_{2} \\
M^{-1}\left(\overline{\mathbf{x}}_{1}\right) C\left(\overline{\mathbf{x}}_{1}, \overline{\mathbf{x}}_{2}\right) \overline{\mathbf{x}}_{2}-M^{-1}\left(\overline{\mathbf{x}}_{1}\right) G\left(\overline{\mathbf{x}}_{1}\right)
\end{array}\right]+} \\
& {\left[\begin{array}{c}
\mathbf{0} \\
M^{-1}\left(\overline{\mathbf{x}}_{1}\right)
\end{array}\right] \mathbf{u} . }
\end{aligned}
$$

As it is very well known, $M^{-1}\left(\bar{x}_{1}\right)$ always exists. Define the following positive function

$$
V(\overline{\mathbf{x}})=\frac{1}{2} K_{E} \bar{\varepsilon}(\overline{\mathbf{x}})^{2}+\frac{1}{2} \overline{\mathbf{x}}^{T}\left[\begin{array}{ll}
P_{11} & P_{12} \\
P_{21} & P_{22}
\end{array}\right] \overline{\mathbf{x}}
$$

where $\overline{\mathbf{x}}=\left[\begin{array}{ll}\overline{\mathbf{x}}_{1} & \overline{\mathbf{x}}_{2}\end{array}\right]^{T}, P_{12 \times 12}$ represents a strictly symmetric and positive definite matrix and its elements $P_{11}=P_{11}^{T}>0$, $P_{12}=P_{21}^{T}>0$ and $P_{22}=P_{22}^{T}>0, K_{E}$ defines a strictly positive definite constant and $\bar{\varepsilon}(\overline{\mathbf{x}})=\varepsilon-\varepsilon_{e q}$ signifies the energy error function. Thus, the total energy of the quadrotor can be described using its Lagrangian equation by

$$
\varepsilon=\frac{1}{2} \overline{\mathbf{x}}_{2}^{T} M\left(\overline{\mathbf{x}}_{1}\right) \overline{\mathbf{x}}_{2}+U\left(\overline{\mathbf{x}}_{1}\right)
$$

where $U=m g z$ is the potential energy of the vehicle. In term of the error function, the above yields

$$
\bar{\varepsilon}(\overline{\mathbf{x}})=\frac{1}{2} \overline{\mathbf{x}}_{2}^{T} M\left(\overline{\mathbf{x}}_{1}\right) \overline{\mathbf{x}}_{2}+U\left(\overline{\mathbf{x}}_{1}\right) .
$$

Differentiating (12) along to the trajectories of system (9), we obtain

$\dot{\bar{\varepsilon}}(\overline{\mathbf{x}})=\frac{1}{2} \overline{\mathbf{x}}_{2}^{T} M\left(\overline{\mathbf{x}}_{1}\right) \dot{\mathbf{\mathbf { x }}}_{2}+\frac{1}{2} \dot{\dot{\mathbf{x}}}_{2}^{T} M\left(\overline{\mathbf{x}}_{1}\right) \overline{\mathbf{x}}_{2}+\frac{1}{2} \overline{\mathbf{x}}_{2}^{T} \dot{M}\left(\overline{\mathbf{x}}_{1}\right) \overline{\mathbf{x}}_{2}+\overline{\mathbf{x}}_{2}^{T} G\left(\overline{\mathbf{x}}_{1}\right)$

Notice that $M=M^{T}$ and $M>0$ thus, the above yields

$$
\dot{\bar{\varepsilon}}(\overline{\mathbf{x}})=\overline{\mathbf{x}}_{2}^{T} M\left(\overline{\mathbf{x}}_{1}\right) \dot{\overline{\mathbf{x}}}_{2}+\frac{1}{2} \overline{\mathbf{x}}_{2}^{T} \dot{M}\left(\overline{\mathbf{x}}_{1}\right) \overline{\mathbf{x}}_{2}+\overline{\mathbf{x}}_{2}^{T} G\left(\overline{\mathbf{x}}_{1}\right) .
$$

Introducing (9) into (13), it follows that

$$
\dot{\bar{\varepsilon}}(\overline{\mathbf{x}})=\overline{\mathbf{x}}_{2}^{T}\left(\mathbf{u}-C\left(\overline{\mathbf{x}}_{1}, \overline{\mathbf{x}}_{2}\right) \overline{\mathbf{x}}_{2}-G\left(\overline{\mathbf{x}}_{1}\right)\right)+\frac{1}{2} \overline{\mathbf{x}}_{2}^{T} \dot{M}\left(\overline{\mathbf{x}}_{1}\right) \overline{\mathbf{x}}_{2}+\overline{\mathbf{x}}_{2}^{T} G\left(\overline{\mathbf{x}}_{1}\right)
$$

and taking into account that the skew-symmetric property satisfies the relationship $\overline{\mathbf{x}}_{2}^{T}\left[\frac{1}{2} \dot{M}\left(\overline{\mathbf{x}}_{1}\right)-C\left(\overline{\mathbf{x}}_{1}, \overline{\mathbf{x}}_{2}\right)\right] \overline{\mathbf{x}}_{2}=0$, we obtain

$$
\dot{\bar{\varepsilon}}(\overline{\mathbf{x}})=\overline{\mathbf{x}}_{2}^{T} \mathbf{u}
$$

Integrating (14),

$$
\int_{0}^{t} \dot{\bar{\varepsilon}}(\overline{\mathbf{x}}) d t=\int_{0}^{t} \overline{\mathbf{x}}_{2}^{T} \mathbf{u} d t \Rightarrow \dot{\bar{\varepsilon}}(\overline{\mathbf{x}})-\dot{\bar{\varepsilon}}(\mathbf{0})=\int_{0}^{t} \overline{\mathbf{x}}_{2}^{T} \mathbf{u} d t,
$$

which implies that the system verifies the passivity property.

On the other hand, differentiating (10) along the trajectories of (8), and introducing the passivity property it follows

$$
\begin{aligned}
\dot{V}(\overline{\mathbf{x}})= & K_{E} \bar{\varepsilon}(\overline{\mathbf{x}}) \dot{\bar{\varepsilon}}(\overline{\mathbf{x}})+\frac{1}{2} \overline{\mathbf{x}}^{T}\left[\begin{array}{ll}
P_{11} & P_{12} \\
P_{21} & P_{22}
\end{array}\right] \dot{\overline{\mathbf{x}}}+ \\
& \frac{1}{2} \dot{\mathbf{x}}^{T}\left[\begin{array}{ll}
P_{11} & P_{12} \\
P_{21} & P_{22}
\end{array}\right] \overline{\mathbf{x}} \\
= & K_{E} \bar{\varepsilon}(\overline{\mathbf{x}}) \overline{\mathbf{x}}_{2}^{T} \mathbf{u}+\overline{\mathbf{x}}_{1}^{T} P_{11} \overline{\mathbf{x}}_{2}+\overline{\mathbf{x}}_{2}^{T} P_{21} \overline{\mathbf{x}}_{2} \\
& +\overline{\mathbf{x}}_{1}^{T} P_{12} \dot{\overline{\mathbf{x}}}_{2}+\overline{\mathbf{x}}_{2}^{T} P_{22} \dot{\overline{\mathbf{x}}}_{2} .
\end{aligned}
$$

Introducing (8) into above, it yields

$$
\begin{aligned}
\dot{V}(\overline{\mathbf{x}})= & \left(\overline{\mathbf{x}}_{1}^{T} P_{12}+\overline{\mathbf{x}}_{2}^{T} P_{22}\right) M\left(\overline{\mathbf{x}}_{1}\right)^{-1}\left(\mathbf{u}-C\left(\overline{\mathbf{x}}_{1}, \overline{\mathbf{x}}_{2}\right) \overline{\mathbf{x}}_{2}-G\left(\overline{\mathbf{x}}_{1}\right)\right) \\
& +K_{E} \bar{\varepsilon}(\overline{\mathbf{x}}) \overline{\mathbf{x}}_{2}^{T} \mathbf{u}+\overline{\mathbf{x}}_{1}^{T} P_{11} \overline{\mathbf{x}}_{2}+\overline{\mathbf{x}}_{2}^{T} P_{21} \overline{\mathbf{x}}_{2}
\end{aligned}
$$

Now, applying dynamic programming

$$
\min _{\mathbf{u}}\left\{\left.\frac{d V(\overline{\mathbf{x}})}{d t}\right|_{(9)}+\mathbf{f}_{\mathbf{0}}(\overline{\mathbf{x}}, \mathbf{u})\right\}
$$

with $\mathbf{f}_{\mathbf{0}}(\overline{\mathbf{x}}, \mathbf{u})=\overline{\mathbf{x}}^{T} Q \overline{\mathbf{x}}+\mathbf{u}^{T} R \mathbf{u}$ and using (15), we get

$$
\min _{\mathbf{u}}\left\{\begin{array}{c}
\left(\overline{\mathbf{x}}_{1}^{T} P_{12}+\overline{\mathbf{x}}_{2}^{T} P_{22}\right) M\left(\overline{\mathbf{x}}_{1}\right)^{-1}\left(\mathbf{u}-C\left(\overline{\mathbf{x}}_{1}, \overline{\mathbf{x}}_{2}\right) \overline{\mathbf{x}}_{2}-G\left(\overline{\mathbf{x}}_{1}\right)\right) \\
+K_{E} \bar{\varepsilon}(\overline{\mathbf{x}}) \overline{\mathbf{x}}_{2}^{T} \mathbf{u}+\overline{\mathbf{x}}_{1}^{T} P_{11} \overline{\mathbf{x}}_{2}+\overline{\mathbf{x}}_{2}^{T} P_{21} \overline{\mathbf{x}}_{2}+\overline{\mathbf{x}}^{T} Q \overline{\mathbf{x}} \\
+\frac{1}{2} \mathbf{u}^{T} R \mathbf{u} .
\end{array}\right\}
$$


Note that this function, is a convex function with respect to u, then, using Theorem 2,

$$
\begin{aligned}
\frac{\partial}{\partial \mathbf{u}}\left\{K_{E} \bar{\varepsilon}(\overline{\mathbf{x}}) \overline{\mathbf{x}}_{2}^{T} \mathbf{u}+\overline{\mathbf{x}}_{1}^{T} P_{11} \overline{\mathbf{x}}_{2}+\overline{\mathbf{x}}_{2}^{T} P_{21} \overline{\mathbf{x}}_{2}\right. & \\
+\left(\overline{\mathbf{x}}_{1}^{T} P_{12}+\overline{\mathbf{x}}_{2}^{T} P_{22}\right) M\left(\overline{\mathbf{x}}_{1}\right)^{-1}\left(\mathbf{u}-C\left(\overline{\mathbf{x}}_{1}, \overline{\mathbf{x}}_{2}\right) \overline{\mathbf{x}}_{2}-G\left(\overline{\mathbf{x}}_{1}\right)\right) & \\
\left.+\overline{\mathbf{x}}^{T} Q \overline{\mathbf{x}}+\frac{1}{2} \mathbf{u}^{T} R \mathbf{u}\right\} & =0 \\
K_{E} \bar{\varepsilon}(\overline{\mathbf{x}}) \overline{\mathbf{x}}_{2}+M^{-1}\left(\overline{\mathbf{x}}_{1}\right)\left(P_{12} \overline{\mathbf{x}}_{1}+P_{22} \overline{\mathbf{x}}_{2}\right)+R \mathbf{u} & =0
\end{aligned}
$$

Since the function $V(\overline{\mathbf{x}})$ is only an approximation of the Bellman function $V^{*}(\mathbf{x}(t))$, a sub-optimal control law for the quadrotor flying vehicle is obtained

$$
\mathbf{u}=-R^{-1}\left\{K_{E} \bar{\varepsilon}(\overline{\mathbf{x}}) \overline{\mathbf{x}}_{2}+M^{-1}\left(\overline{\mathbf{x}}_{1}\right)\left(P_{12} \overline{\mathbf{x}}_{1}+P_{22} \overline{\mathbf{x}}_{2}\right)\right\}
$$

The previous result can be summarized in the following proposition.

Proposition 1: Consider the nonlinear model of the quadrotor helicopter given by

$\dot{\overline{\mathbf{x}}}=\left[\begin{array}{c}\overline{\mathbf{x}}_{2} \\ M^{-1}\left(\overline{\mathbf{x}}_{1}\right) C\left(\overline{\mathbf{x}}_{1}, \overline{\mathbf{x}}_{2}\right) \overline{\mathbf{x}}_{2}-M^{-1}\left(\overline{\mathbf{x}}_{1}\right) G\left(\overline{\mathbf{x}}_{1}\right)\end{array}\right]+\left[\begin{array}{c}\mathbf{0} \\ M^{-1}\left(\overline{\mathbf{x}}_{1}\right)\end{array}\right] \mathbf{u}$.

and the function $V(\mathbf{x})$ given by (10). Then, a sub-optimal control for this system is given by

$$
\mathbf{u}=-R^{-1}\left\{K_{E} \bar{\varepsilon}(\overline{\mathbf{x}}) \overline{\mathbf{x}}_{2}+M^{-1}\left(\overline{\mathbf{x}}_{1}\right)\left(P_{12} \overline{\mathbf{x}}_{1}+P_{22} \overline{\mathbf{x}}_{2}\right)\right\}
$$

Remark 1: The control law given by (18) holds for fully actuated systems. Nevertheless, it is possible to apply it to the quadrotor model given by (1) since it can be rewritten in the standard form $\dot{\mathbf{x}}=f(\mathbf{x})+g(\mathbf{x}) \mathbf{u}$ as (9).

\section{NUMERICAL VALIDATION}

To validate the performance of the proposed control law (17) in closed loop, some simulations have been carried out. In order to find the appropriate values for the matrix $P_{12}$ and $P_{22}$, the system is linearized and then, the wellknown algebraic Riccati equation solved. Hence, it follows that (1) can be rewritten in the standard form (9). Its linear approximation can be expressed as: $\dot{\mathbf{x}}=A \mathbf{x}+B \mathbf{u}$ where $A=\frac{\partial f(\mathbf{x})}{\partial \mathbf{x}}, B=\frac{\partial g(\mathbf{x})}{\partial \mathbf{u}}, \mathbf{x}=\mathbf{q}$ and $\mathbf{u}=\left[\begin{array}{llll}u & \tau_{\psi} & \tau_{\theta} & \tau_{\phi}\end{array}\right]^{T}$. The values of the matrix $Q$ and $R$ were chosen as

$$
\begin{gathered}
Q=\operatorname{diag}\{5,5,250,5,50,50,5,5,5,5,5,5\}, \\
R=\operatorname{diag}\{10000,20000,40000,20000\}
\end{gathered}
$$

Finally, solving the Riccati equation, the values of $P_{12}$ and $P_{22}$ are

$$
\begin{gathered}
P_{12}=\left[\begin{array}{cccccc}
53 & 0 & 0 & 0 & -447 & 0 \\
0 & 45 & 0 & 0 & 0 & 316 \\
0 & 0 & 1581 & 0 & 0 & 0 \\
0 & 0 & 0 & 316 & 0 & 0 \\
-2783 & 0 & 0 & 0 & 46755 & 0 \\
0 & 1734 & 0 & 0 & 0 & 27970
\end{array}\right] \\
P_{22}=\left[\begin{array}{cccccc}
182 & 0 & 0 & 0 & -2113 & 0 \\
0 & 143 & 0 & 0 & 0 & 1380 \\
0 & 0 & 5628 & 0 & 0 & 0 \\
0 & 0 & 0 & 3571 & 0 & 0 \\
-2113 & 0 & 0 & 0 & 61160 & 0 \\
0 & 1380 & 0 & 0 & 0 & 33450
\end{array}\right] .
\end{gathered}
$$

The following figures illustrate the performance of the proposed control strategy. The numerical validation was designed in such way that it can represent real applications. We divided the simulations in two cases. In the first case, we supposed that the quadrotor has to do a building inspection with different values of altitude. Then, the quadrotor needs to realize a square of $30 \mathrm{~m}$ in each side and it changes its altitude in the following form

$$
z_{d}=\left\{\begin{array}{cc}
20 \mathrm{~m} & t \leq 70 \mathrm{~s} \\
40 \mathrm{~m} & 70 \mathrm{~s}<t \leq 110 \mathrm{~s} \\
30 \mathrm{~m} & 110 \mathrm{~s}<t \leq 180 \mathrm{~s} \\
0 \mathrm{~m} & 180 \mathrm{~s} \leq 200 \mathrm{~s}
\end{array}\right.
$$

The desired values for the angles were $\psi_{d}=\theta_{d}=\phi_{d}=$ 0 . In Figures $2-4$, we introduce the state and control responses when applying the proposed controller. Notice in these figures the well performance of the closed-loop system. Observe in Figure 3 that the angles in pitch and roll change when the vehicle realize longitudinal and lateral movements. In this figure, we can also see that the yaw response (solid line) does not change.

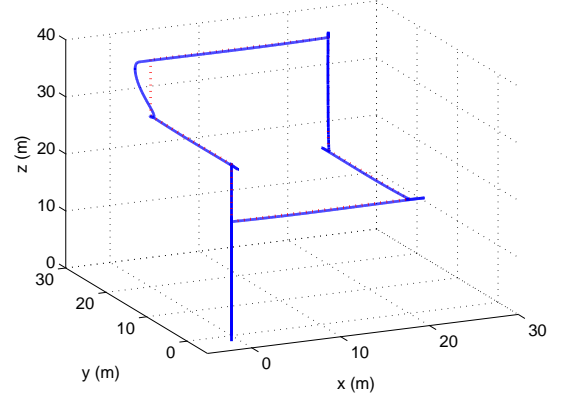

Fig. 2. Position response of the quadrotor using the proposed control law (17).

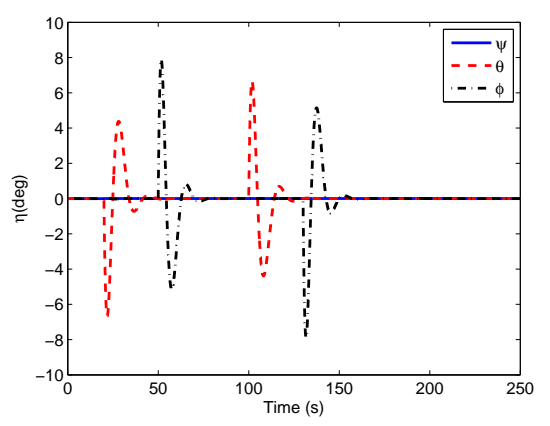

Fig. 3. Attitude behavior of the quadrotor using the proposed passivity algorithms.

Similarly in Figure 4, notice that the amplitude of the control inputs is acceptable. This figure can be compared with Figure 7 where a feedback controller is used, i.e $\mathbf{u}=$ $-K \overline{\mathbf{x}}$. It can be observed that when using this feedback controller the responses of the control inputs are higher than 
with the proposed controller. Then, it is not always possible to apply them in a real application because of their high amplitude. The state responses of the feedback controller are depicted in Figures $5-6$. We use the well known Ackermann's formula to find the values of $K$. Notice in these figures that, even if the vehicle follows the desired trajectory, some errors in the position can be observed, see Figure 5.
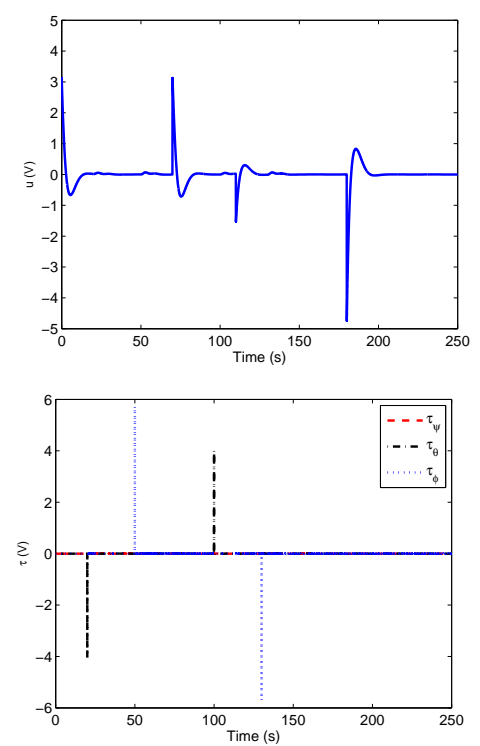

Fig. 4. Control inputs responses of the quadrotor using (17).

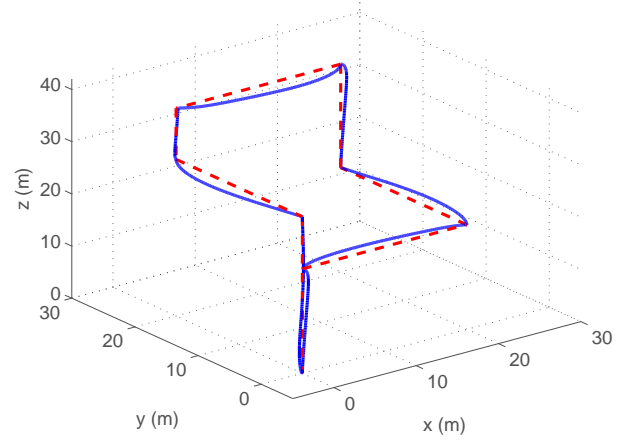

Fig. 5. Position response of the quadrotor using a linear feedback control. In this figure the dashed line is the reference.

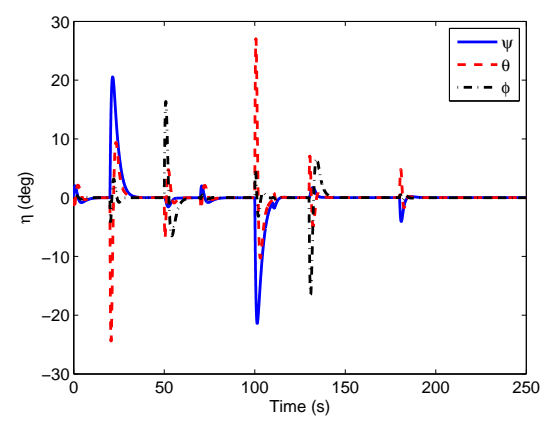

Fig. 6. Orientation behavior of the quadrotor using a linear feedback control
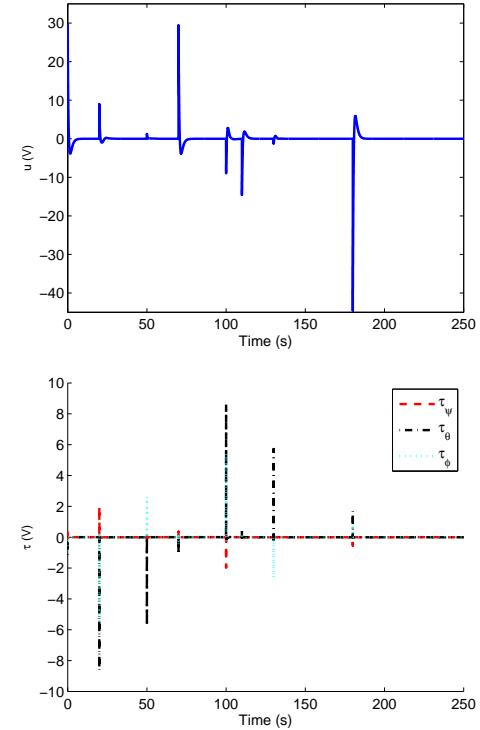

Fig. 7. Control inputs responses of the quadrotor using a linear feedback control

Likewise, the attitude behavior is degraded and the angles responses are bigger than when using the passivity control strategy. Notice also in this figure that there are small changes in the yaw angle whereas it is not the case when using the proposed algorithm. Finally, we can remark that the control inputs of the nonlinear control are much smaller compared with the control inputs of the linear controller.

In practical situations, when the quadrotor operates, disturbances are always present (wind for example). The objective, for the second case, was to stabilize the quadrotor in a desired position in presence of lateral wind, $V_{w}$. The simulations were carried out considering the following assumptions:

- The quadrotor aircraft is stabilized in the desired position with small disturbances. Then, $\forall t>T_{1}$ the helicopter is in hover.

- A lateral wind gust is applied as Figure 8 shows.

- The initial conditions were $\xi(0)=(0,0,0) \mathrm{m}$ and $\eta(0)=(0,0,0)^{\circ}$ while the desired positions were $\xi_{d}=$ $(20,20,20) \mathrm{m}$ and $\eta_{d}=(0,0,0)^{\circ}$.

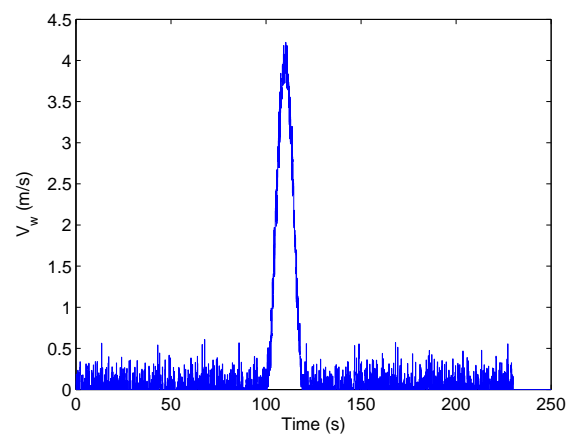

Fig. 8. Signal disturbance. 
The quadrotor performance was also compared with another control approach. For this case, the Linear Quadratic Regulator (LQR) was used. For both control laws, the values for the matrix $Q$ are the same that the ones used previously. Instead the values for the matrix $R$ were modified to $R=$ $\operatorname{diag}\{10000,10000,10000,10000\}$ in order to improve the behavior.

The results are introduced in Figures 9 - 11. Notice in Figure 9 that, both controllers have similar behavior unless when the perturbation appears. Observe in this figure that the LQR controller is not capable to remain close to the desired position of the vehicle. Similarly, in Figures 10 and 11, we can remark the similar performance of both algorithms except also when the disturbance is applied.

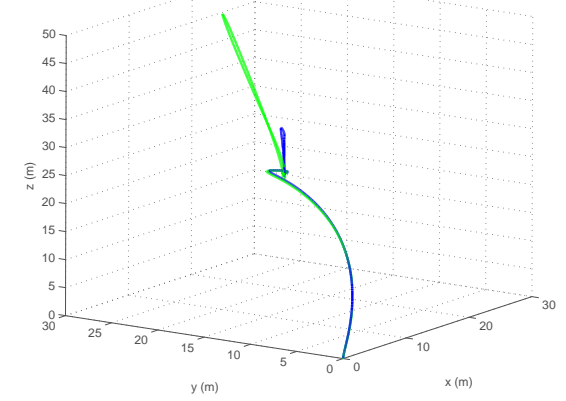

Fig. 9. 3D position responses of the aerial vehicle in presence of lateral wind gust. Solid line represents the behavior using the proposed control scheme while dashed line (green) represents the LQR algorithm.

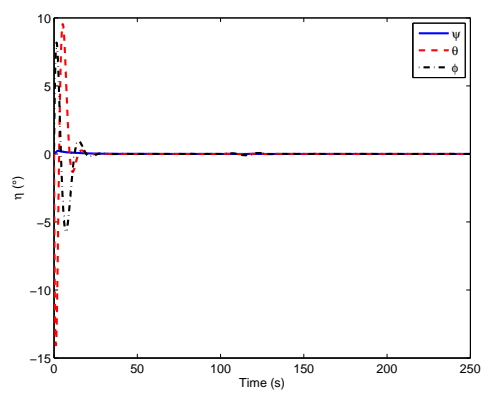

Fig. 10. Attitude performance of the quadrotor in presence of lateral wind gust when using (17).

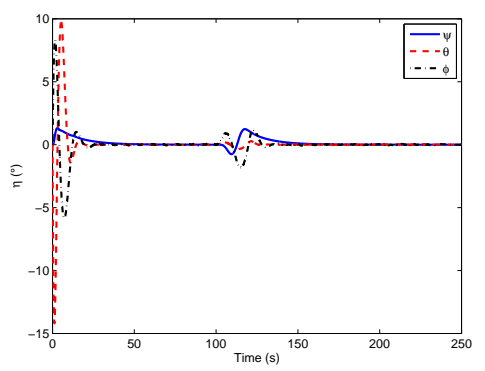

Fig. 11. Attitude performance of the quadrotor in presence of lateral wind gust when using the LQR control laws.

\section{CONCLUSion}

A new energy-based control algorithm for the stabilization of a quadrotor was introduced in this paper. The main contribution is to take advantage of the passivity property of the system and the use of Dynamic Programming approach. Indeed, an energy function and a quadratic term have been utilized in order to design a sub-optimal control law for stabilization of the quadrotor system. The closed-loop behavior using the proposed algorithm has been compared with those when using a linear feedback controller and a LQR algorithm. Simulations have shown that the performance of the proposed control design is satisfactory also in presence of a wind gust perturbation. Future work includes the experimental tests in a quadrotor platform.

\section{REFERENCES}

[1] Gabriel M. Hoffmann, Steven L. Waslander, and Claire J. Tomlin, "Distributed Cooperative Search using Information-Theoretic Costs for Particle Filters, with Quadrotor Applications", AIAA Guidance, Navigation, and Control Conference and Exhibit, Keystone, Colorado, USA, 21 - 24 August 2006.

[2] Gabriel M. Hoffmann, Haomiao Huang, Steven L. Waslander and Claire J. Tomlin, "Quadrotor Helicopter Flight Dynamics and Control: Theory and Experiment", AIAA Guidance, Navigation and Control Conference and Exhibit, Hilton Head, South Carolina, USA, 20 - 23 August 2007.

[3] Raffo, G. V., Ortega, M. G., and Rubio, F. R., "An integral predictive/nonlinear $H_{\infty}$ control structure for a quadrotor helicoper". Automatica, Vol. 46, No. 1, Jan 2010, pp. 29 - 39.

[4] Castillo, P., Lozano, R. and Dzul, A., "Stabilization of a minirotorcraft having four rotors". Proceedings of IEEE/RSJ International Conference on Intelligent Robots and Systems, Vol. 3, Sendai, Japan, 2004, pp. $2693-2698$

[5] Dierks, T. and Jagannathan, S., "Output Feedback Control of a Quadrotor UAV Using Neural Networks". IEEE Transactions on Neural Networks, Vol.21, No.1, Jan. 2010, pp. 50 - 66.

[6] Voss, H., " Nonlinear Control of a Quadrotor Micro-UAV using Feedback-Linearization". Proceedings of IEEE International Conference on Mechatronics. Malaga, Spain, April 2009, pp. 1- 6.

[7] Madani,T. and Benallegue, A., "Control of a quadrotor mini-helicopter via full state backstepping technique", Proceedings of the 45th IEEE Conference on Decision and Control, San Diego, CA, USA, December 2006.

[8] Kottenstette, N. and Porter, J., "Digital passive attitude and altitude control schemes for quadrotor aircraft", IEEE International Conference on Control and Automation (ICCA'09), Christchurch, New Zealand, pp.1761-1768, December 9-11, 2009.

[9] Mokhtari, A., Benallegue, A. and Daachi, B., "Robust Feedback linearization and $G H_{\infty}$ controller for a quadrotor unmanned aerial vehicle", IEEE/RSJ International Conference on Intelligent Robots and Systems, IROS, Edmonton, Alberta, Canada, August 2-6, 2005.

[10] Alexis, K., Nikolakopoulos, G. and Tzes, A., "Constrained optimal attitude control of a quadrotor helicopter subject to wind-gusts: Experimental studies", American Control Conference (ACC), Baltimore, Maryland, USA, pp.4451-4455, June 30-July 2, 2010.

[11] Ordaz-Oliver, P., Santos-Sanchez, O. and Lopez-Morales V., "On the sub-optimal feedback control law synthesis of underactuated systems". International Journal of Innovative Computing, Information and Control, Vol. 5, 9., September 2009, pp 2791-2807.

[12] Ordaz-Oliver, J. P., Santos-Sánchez, O. J., López-Morales, V. Toward a generalized sub-optimal control method of underactuated systems, Optimal Control Applications and Methods, Vol. 33, 3, pp 338-351, 2012.

[13] Castillo, P., Lozano, R. and Dzul, A., "Modelling and Control of Mini-Flying Machines", Advances in Industrial Control, 1st Ed., 2005, London UK: Springer-Verlag, pp. 39 - 59, ISBN: 978-1-85233-957-9.

[14] Lee E. and Markus L., Foundations of optimal control theory. Wiley, New York, 1967.

[15] Dyer P. and McReynolds S.R., The computation and theory of optimal control. Academic Press, New York, 1970. 